\title{
Refined Estimates and Generalizations of Inequalities Related to the Arctangent Function and Shafer's Inequality
}

\author{
Branko Maleševićc (D, Marija Rašajski, and Tatjana Lutovac \\ University of Belgrade, School of Electrical Engineering, Department of Applied Mathematics, Serbia \\ Correspondence should be addressed to Branko Malešević; branko.malesevic@etf.bg.ac.rs
}

Received 10 November 2017; Accepted 11 September 2018; Published 10 October 2018

Academic Editor: Luis M. López-Ochoa

Copyright (C) 2018 Branko Malešević et al. This is an open access article distributed under the Creative Commons Attribution License, which permits unrestricted use, distribution, and reproduction in any medium, provided the original work is properly cited.

We give some sharper refinements and generalizations of inequalities related to Shafer's inequality for the arctangent function, stated in Theorems 1,2, and 4 in Mortici and Srivastava, 2014, by C. Mortici and H.M. Srivastava.

\section{Introduction}

Inverse trigonometric functions play an important role and have many applications in engineering [1-4]. In particular, the arctangent function and various related inequalities have been studied and effectively applied to problems in fundamental sciences and many areas of engineering, such as electronics, mechanics, and aeronautics [3, 5, 6]; see also [7].

Various approximations of the arctangent function can be found in $[4-6,8-21]$; see also $[22,23]$. One of the inequalities that attracted attention of many authors is Shafer's inequality [9]:

$$
\frac{3 x}{1+2 \sqrt{1+x^{2}}}<\arctan x
$$

which holds for $x>0$; see also [10-12].

Recently, in [8], Mortici and Srivastava proved the following results, cited here as Statements 1,2, and 3, related to the above inequality. These results are the starting point of our research.

Statement 1 (Theorem 1, [8]). For every $x>0$, the following two-sided inequality holds:

$$
\begin{aligned}
\frac{3 x}{1+2 \sqrt{1+x^{2}}}+a(x) & <\arctan x \\
& <\frac{3 x}{1+2 \sqrt{1+x^{2}}}+b(x),
\end{aligned}
$$

where $a(x)=(1 / 180) x^{5}-(13 / 1512) x^{7}$ and $b(x)=(1 / 180) x^{5}$.
Statement 2 (Theorem 2, [8]). For every $x>0$, it is asserted that

$$
\frac{3 x+c(x)}{1+2 \sqrt{1+x^{2}}}<\arctan x<\frac{3 x+d(x)}{1+2 \sqrt{1+x^{2}}}
$$

where $c(x)=(1 / 60) x^{5}-(17 / 840) x^{7}$ and $d(x)=(1 / 60) x^{5}$.

Statement 3 (Theorem 4, [8]). For every $x>0$, it is asserted that

$$
-\frac{1}{12} x^{3}<\arctan x-\frac{2 x}{1+\sqrt{1+x^{2}}}<-\frac{1}{12} x^{3}+\frac{3}{40} x^{5} .
$$

The main results of this paper are refined estimates and generalizations of the inequalities given in Statements 1,2 , and 3. Although inequalities (2), (3), and (4) hold for $x>0$, considering them in a neighborhood of zero is of primary importance, as noted in [8].

\section{Main Results}

First, let us recall some well-known power series expansions that will be used in our proofs.

For $|x| \leq 1$,

$$
\arctan x=\sum_{m=0}^{\infty}(-1)^{m} A(m) x^{2 m+1},
$$


where

$$
A(m)=\frac{1}{2 m+1} .
$$

For $|x| \leq 1$,

$$
\sqrt{1+x^{2}}=1+\sum_{m=0}^{\infty}(-1)^{m} K(m) x^{2 m+2}
$$

where

$$
K(m)=\frac{(2 m) !}{m !(m+1) ! 2^{2 m+1}} .
$$

The following power series expansion holds:

$$
\frac{3 x}{1+2 \sqrt{1+x^{2}}}=\sum_{m=0}^{\infty} B(m) x^{2 m+1},
$$

where $|x| \leq \sqrt{3} / 2$, with $B(0)=1$ and $B(1)=-1 / 3$, and for $m \geq 2$,

$$
B(m)=\frac{(-1)^{m} 4^{m-1}}{3^{m}}\left(1-8 \sum_{i=2}^{m} \frac{(2 i-2) !}{(i-1) ! i ! 2^{2 i-1}}\left(\frac{3}{4}\right)^{i}\right) .
$$

Power series coefficients are calculated by applying Cauchy's product to the power series expansions arising from the following transformation of the corresponding function:

$$
\frac{3 x}{1+2 \sqrt{1+x^{2}}}=-\frac{x\left(1-2 \sqrt{1+x^{2}}\right)}{1+(4 / 3) x^{2}} .
$$

It is easy to prove that sequence $\{B(m)\}_{m \in N_{0}}$ for $m \geq 1$ satisfies the recurrence equation:

$$
\begin{aligned}
B(m+1)+\frac{4}{3} B(m) & =(-1)^{m} \frac{(2 m-1) !}{2^{2 m-1}(m-1) !(m+1) !} \\
& =2(-1)^{m} \frac{(2 m-1) ! !}{(2 m+2) ! !} .
\end{aligned}
$$

2.1. Refinements of the Inequalities in Statement 1. Before we proceed to Theorem 9, which represents an improvement and generalization of Statement 1, we need the following lemmas.

Lemma 4. Let $\beta(0)=1, \beta(1)=-1 / 3$, and $\beta(m)=$ $\left(3(-1)^{m} / 2^{2 m+1}\right) \sum_{k=0}^{\infty}((2 k+2 m-1) ! /(k+m-1) !(k+m+$ $1) !)(3 / 16)^{k}$, for $m \geq 2$. The sequence $\{\beta(m)\}_{m \in N_{0}}$ for $m \geq 1$ satisfies the recurrence relation (12).

Proof. In the proof of this lemma we use the Wilf- Zeilberger method [24-26]. (The same approach we used in [27].)

The assertion is obviously true for $m=1$.

Let $m \geq 2$ and

$$
\begin{aligned}
g(k, m) & =\frac{(2 k+2 m-1) !}{(k+m-1) !(k+m+1) !} \text { and } \\
\phi(m) & =\frac{3(-1)^{m}}{2^{2 m+1}} .
\end{aligned}
$$

Then we have

$$
\beta(m)=\phi(m) \sum_{k=0}^{\infty} \mathrm{g}(k, m)\left(\frac{3}{16}\right)^{k} .
$$

Further we have

$$
\begin{aligned}
& \beta(m+1)+\frac{4}{3} \beta(m) \\
& =\sum_{k=0}^{\infty}\left(\phi(m+1) g(k, m+1)+\frac{4}{3} \phi(m) g(k, m)\right) \\
& \cdot\left(\frac{3}{16}\right)^{k}=\frac{(-1)^{m}}{2^{(2 m-1)}} \\
& \cdot \sum_{k=0}^{\infty} \frac{(2 k+2 m+13)(2 k+2 m-1) !}{(8 k+8 m+16)(k+m-1) !(k+m+1) !} \\
& \cdot\left(\frac{3}{16}\right)^{k} .
\end{aligned}
$$

Consider now the sequence $\{\mathrm{S}(m)\}_{m \in N, m \geq 2}$, where

$$
\begin{aligned}
& \mathrm{S}(m)=\sum_{k=0}^{m-1} \mathrm{~F}(m, k) \text { and } \\
& \mathrm{F}(m, k) \\
& \quad=\frac{(2 m+2 k+13)(2 m+2 k-1) !}{(8 m+8 k+16)(m+k-1) !(m+k+1) !}\left(\frac{3}{16}\right)^{k} .
\end{aligned}
$$

Consider the function (an algorithm for determining function $\mathrm{G}(m, k)$ for a given function $\mathrm{F}(m, k)$ is described in [24]. Note that the pair of discrete functions $(\mathrm{F}(m, k), \mathrm{G}(m, k))$ is the so-called Wilf- Zeilberger pair)

$$
\begin{aligned}
& \mathrm{G}(m, k) \\
& =\frac{(-8 k-8 m-16)(2 k+2 m-1) !}{(8 k+8 m+16)(k+m-1) !(k+m+1) !}\left(\frac{3}{16}\right)^{k},
\end{aligned}
$$

where $m \in N_{0}$ and $k \in N_{0}$. It is not hard to verify that functions $\mathrm{F}(m, k)$ and $\mathrm{G}(m, k)$ satisfy the following relation:

$$
\mathrm{F}(m, k)=\mathrm{G}(m, k+1)-\mathrm{G}(m, k) .
$$

If we sum both sides of (18) over all $k \in N_{0}$, we get the following relation:

$$
S(m)=-\mathbf{G}(m, 0) .
$$

Finally, as

$$
\mathrm{G}(m, 0)=-\frac{(2 m-1) !}{(m-1) !(m+1) !}
$$

we have

$$
S(m)=\frac{(2 m-1) !}{(m-1) !(m+1) !} .
$$


Therefore from (15) and (21) we conclude that

$$
\begin{aligned}
\beta(m+1)+\frac{4}{3} \beta(m) & =\frac{(-1)^{m}}{2^{2 m-1}} \cdot S(m) \\
& =\frac{(-1)^{m}}{2^{2 m-1}} \cdot \frac{(2 m-1) !}{(m-1) !(m+1) !} .
\end{aligned}
$$

Corollary 5. Given that the sequences $\{\mathrm{B}(m)\}_{m \in N_{0}}$ and $\{\beta(m)\}_{m \in N_{0}}$ satisfy the same recurrence relation and as they agree for $m=0$ and $m=1$, we conclude that

$$
\mathrm{B}(m)=\beta(m), \quad \text { for } m \in N_{0} \text {. }
$$

Let us introduce the notation:

$$
C(m)=A(m)-B(m),
$$

where $C(0)=C(1)=0$, and for $m \geq 2$ the following holds:

$$
\begin{aligned}
C(m)= & \frac{1}{2 m+1} \\
& -\frac{4^{m-1}}{3^{m}}\left(1-8 \sum_{i=2}^{m} \frac{(2 i-2) !}{(i-1) ! i ! 2^{2 i-1}}\left(\frac{3}{4}\right)^{i}\right) .
\end{aligned}
$$

Thus, we have the power series expansion:

$$
\begin{aligned}
f(x) & =\arctan x-\frac{3 x}{2+\sqrt{1+x^{2}}} \\
& =\sum_{m=0}^{\infty}(-1)^{m} C(m) x^{2 m+1},
\end{aligned}
$$

for $m \in N_{0}$ and $|x| \leq \sqrt{3} / 2$.

Let us introduce the notation:

$$
\begin{aligned}
& \beta^{+}(m) \\
& =\frac{3}{2^{2 m+1}} \sum_{k=0}^{\infty} \frac{(2 k+2 m-1) !}{(k+m-1) !(k+m+1) ! 2^{2 k}}\left(\frac{3}{4}\right)^{k}, \\
& \beta_{1}(m)=\sum_{k=0}^{+\infty} \frac{\prod_{n=0}^{k-1}(m+1 / 2+n)}{\prod_{n=0}^{k-1}(m+2+n)}\left(\frac{3}{4}\right)^{k}, \quad m \in N_{0} \\
& \beta_{1}^{k}(m)=\frac{\prod_{n=0}^{k-1}(m+1 / 2+n)}{\prod_{n=0}^{k-1}(m+2+n)},
\end{aligned}
$$

$m \in N_{0}$.

Lemma 6. For $m \in N_{0}$ the following holds:

$$
\beta^{+}(m)=\frac{3}{2} \frac{(2 m-1) ! !}{(2 m+2) ! !} \beta_{1}(m)
$$

Proof.

$$
\begin{gathered}
\frac{3}{2^{2 m+1}} \frac{(2 m+2 k-1) !}{(m+k-1) !(m+k+1) ! 2^{2 k}}=\frac{3}{2} \frac{1}{(2 m+2) ! !} \\
\cdot \frac{(2 m+2 k-1) ! !}{(m+k+1) !} \frac{(m+1) !}{2^{k}}=\frac{3}{2} \frac{(2 m-1) ! !}{(2 m+2) ! !} \\
\cdot \frac{(2 m+1)(2 m+3) \ldots(2 m+2 k-1)}{(m+1) !(m+2)(m+3) \ldots(m+k+1)} \\
\cdot \frac{(m+1) !}{2^{k}}=\frac{3}{2} \frac{(2 m-1) ! !}{(2 m+2) ! !} \frac{\prod_{n=0}^{k-1}(m+1 / 2+n)}{\prod_{n=0}^{k-1}(m+2+n)} .
\end{gathered}
$$

Lemma 7. For $m \in N_{0}$ the following holds:

$$
\begin{aligned}
\frac{8(m+2)}{2 m+13} & =\sum_{k=0}^{\infty}\left(\frac{m+1 / 2}{m+2}\right)^{k}\left(\frac{3}{4}\right)^{k}<\beta_{1}(m) \\
& <\sum_{k=0}^{\infty}\left(\frac{3}{4}\right)^{k}=4 .
\end{aligned}
$$

Proof. The statement immediately follows from the inequalities:

$$
\left(\frac{m+1 / 2}{m+2}\right)^{k}<\beta_{1}^{k}(m)<1 .
$$

Lemma 8. For $m \in N_{0}$ the following holds:

$$
\beta_{1}(m+1)>\beta_{1}(m) \frac{m+2}{m+1 / 2}\left(1-\frac{3}{2} \frac{1}{m+2}\right) .
$$

Proof.

$$
\begin{aligned}
\beta_{1}^{k}(m+1) & =\beta_{1}^{k}(m) \frac{(m+2)(m+1 / 2+k)}{(1 / 2+m)(m+2+k)} \\
& =\beta_{1}^{k}(m) \frac{m+2}{m+1 / 2}\left(1-\frac{3}{2} \frac{1}{m+2+k}\right) \\
& >\beta_{1}^{k}(m) \frac{m+2}{m+1 / 2}\left(1-\frac{3}{2} \frac{1}{m+2}\right) .
\end{aligned}
$$

Theorem 9. For the real analytic function

$$
f(x)=\arctan x-\frac{3 x}{2+\sqrt{1+x^{2}}}
$$

the following inequalities hold for $k \in N$ and $x \in(0, \sqrt{3} / 2]$ :

$$
\begin{gathered}
\sum_{m=0}^{2 k+1}(-1)^{m} C(m) x^{2 m+1}<f(x) \\
<\sum_{m=0}^{2 k}(-1)^{m} C(m) x^{2 m+1} .
\end{gathered}
$$


where $C(0)=C(1)=0$, and for $m \geq 2$ the following holds:

$$
\begin{aligned}
C(m)= & \frac{1}{2 m+1} \\
& -\frac{4^{m-1}}{3^{m}}\left(1-8 \sum_{i=2}^{m} \frac{(2 i-2) !}{(i-1) ! i ! 2^{2 i-1}}\left(\frac{3}{4}\right)^{i}\right) .
\end{aligned}
$$

Proof. We will prove that the sequence $\{C(m)\}_{m \in N_{0}}$ is positive and monotonically decreasing and tends to zero as $m$ tends to infinity. We will use Lemmas 7 and 8.

$$
\begin{aligned}
C(m) & =\frac{1}{2 m+1}-\frac{3}{2} \frac{(2 m-1) ! !}{(2 m+2) ! !} \beta_{1}(m) \\
& >\frac{1}{2 m+1}-4 \frac{3}{2} \frac{(2 m-1) ! !}{(2 m+2) ! !} \\
& =\frac{1}{2 m+1}\left(1-6 \frac{(2 m+1) ! !}{(2 m+2) ! !}\right)
\end{aligned}
$$

It is easy to verify that $6((2 m+1) ! ! /(2 m+2) !$ ! $)<1$ for $m \geq 11$; therefore $C(m)>0$. Let us note that $0<C(m)<1 /(2 m+1)$, so we can conclude that $\lim _{m \rightarrow+\infty} C(m)=0$.

Let us now prove that $\{C(m)\}_{m \in N_{0}}$ is a monotonically decreasing sequence.

$$
\begin{aligned}
C & (m+1)-C(m)=\frac{-2}{(2 m+1)(2 m+3)}-\frac{3}{2} \\
& \cdot \frac{(2 m+1) ! !}{(2 m+4) ! !} \beta_{1}(m+1)+\frac{3}{2} \frac{(2 m-1) ! !}{(2 m+2) ! !} \beta_{1}(m) \\
& =\frac{-2}{(2 m+1)(2 m+3)}+\frac{3}{2} \frac{(2 m-1) ! !}{(2 m+2) ! !}\left(\beta_{1}(m)\right. \\
& \left.-\frac{2 m+1}{2 m+4} \beta_{1}(m+1)\right)<\frac{-2}{(2 m+1)(2 m+3)}+\frac{3}{2} \\
& \cdot \frac{(2 m-1) ! !}{(2 m+2) ! !}\left(\beta_{1}(m)-\frac{2 m+1}{2 m+4}\right. \\
& \left.\cdot \frac{m+2}{m+1 / 2}\left(1-\frac{3}{2} \frac{1}{m+2}\right) \beta_{1}(m)\right) \\
& =\frac{-2}{(2 m+1)(2 m+3)}+\beta_{1}(m) \frac{9}{4} \\
& \cdot \frac{(2 m-1) ! !}{(m+2)(2 m+2) ! !}<\frac{-2}{(2 m+1)(2 m+3)}+4 \frac{9}{4} \\
& \cdot \frac{(2 m-1) ! !}{(m+2)(2 m+2) ! !}=\frac{-2}{(2 m+1)(2 m+3)}(1 \\
& \left.\frac{(2 m+4) ! !}{(2 m}\right)
\end{aligned}
$$

It is easy to prove that $C(m+1)-C(m)<0$ for $m \geq 8$, i.e., the sequence is monotonically decreasing. Since $\{C(m)\}_{m \in N_{0}}$ is positive for $m \geq 2$ and monotonically decreasing (for $m \geq$ 8 ) and tends to zero, the same holds true for the sequence $\left\{C(m) x^{2 m+1}\right\}_{m \in N_{0}}$ for a fixed $x \in(0, \sqrt{3} / 2]$ (noting that it is decreasing for $m \geq 3$ ), so we can apply Leibniz's theorem for alternating series [28], thus proving the claim of Theorem 9:

$$
\begin{aligned}
& \sum_{m=0}^{2 k+1}(-1)^{m} C(m) x^{2 m+1}<f(x) \\
& \quad<\sum_{m=0}^{2 k}(-1)^{m} C(m) x^{2 m+1}, \quad k \in N .
\end{aligned}
$$

Examples. For $k=1$ and $x \in(0, \sqrt{3} / 2]$ we get Statement 1 .

For $k=2$ and $x \in(0, \sqrt{3} / 2]$,

$$
\begin{gathered}
\frac{x^{5}}{180}-\frac{13 x^{7}}{1512}+\frac{53 x^{9}}{5184}-\frac{3791 x^{11}}{342144} \\
<\arctan x-\frac{3 x}{1+2 \sqrt{1+x^{2}}} \\
<\frac{x^{5}}{180}-\frac{13 x^{7}}{1512}+\frac{53 x^{9}}{5184}
\end{gathered}
$$

For $k=3$ and $x \in(0, \sqrt{3} / 2]$,

$$
\begin{aligned}
& \frac{x^{5}}{180}-\frac{13 x^{7}}{1512}+\frac{53 x^{9}}{5184}-\frac{3791 x^{11}}{342144}+\frac{55801 x^{13}}{4852224} \\
& -\frac{130591 x^{15}}{11197440}<\arctan x-\frac{3 x}{1+2 \sqrt{1+x^{2}}}<\frac{x^{5}}{180} \\
& -\frac{13 x^{7}}{1512}+\frac{53 x^{9}}{5184}-\frac{3791 x^{11}}{342144}+\frac{55801 x^{13}}{4852224},
\end{aligned}
$$

etc.

2.2. Refinements of the Inequalities in Statement 2. We propose the following improvement and generalization of Statement 2.

Theorem 10. For every $x \in(0,1]$ and $k \in N$, it is asserted that

$$
\begin{gathered}
\frac{3 x+\sum_{m=2}^{2 k+1}(-1)^{m} E(m) x^{2 m+1}}{1+2 \sqrt{1+x^{2}}}<\arctan x \\
<\frac{3 x+\sum_{m=2}^{2 k}(-1)^{m} E(m) x^{2 m+1}}{1+2 \sqrt{1+x^{2}}}
\end{gathered}
$$

where

$$
\begin{aligned}
E(m) & \\
= & \frac{3}{2 m+1} \\
& -\sum_{i=0}^{m-1} \frac{(2 m-2 i-2) !}{2^{(2 m-2 i-2)}(2 i+1)(m-i-1) !(m-i) !} .
\end{aligned}
$$

Examples. For $x \in(0,1]$ and $k=1$ we get inequality (3) from Statement 2.

For $x \in(0,1]$ and $k \geq 2$ inequality (42) refines inequality (3) from Statement 2 and we have the following new results: 
(i) Taking $k=2$ in (42) gives

$$
\begin{aligned}
& \frac{3 x+(1 / 60) x^{5}-(17 / 840) x^{7}+(139 / 6720) x^{9}-(8947 / 443520) x^{11}}{1+2 \sqrt{1+x^{2}}}<\arctan x \\
& <\frac{3 x+(1 / 60) x^{5}-(17 / 840) x^{7}+(139 / 6720) x^{9}}{1+2 \sqrt{1+x^{2}}} .
\end{aligned}
$$

(ii) Taking $k=3$ in (42) gives

$$
\begin{gathered}
\frac{3 x+(1 / 60) x^{5}-(17 / 840) x^{7}+(139 / 6720) x^{9}-(8947 / 443520) x^{11}+(89279 / 4612608) x^{13}-(851677 / 46126080) x^{15}}{1+2 \sqrt{1+x^{2}}} \\
<\arctan x<\frac{3 x+(1 / 60) x^{5}-(17 / 840) x^{7}+(139 / 6720) x^{9}-(8947 / 443520) x^{11}+(89279 / 4612608) x^{13}}{1+2 \sqrt{1+x^{2}}},
\end{gathered}
$$

etc.

Proof of Theorem 10 . Based on Cauchy's product of power series (7) and (5), the real analytical function,

$$
f(x)=\left(1+2 \sqrt{1+x^{2}}\right) \cdot \arctan x-3 x,
$$

for $x \in(0,1]$ has the following power series:

$$
f(x)=\sum_{m=2}^{\infty}(-1)^{m} E(m) x^{2 m+1},
$$

where

$$
\begin{aligned}
E(m)= & \frac{3}{2 m+1} \\
& -\sum_{i=0}^{m-1} \frac{(2 m-2 i-2) !}{(2 i+1)(m-i-1) !(m-i) ! 2^{(2 m-2 i-2)}} .
\end{aligned}
$$

We aim to show that sequence $\{E(m)\}_{m \in N, m \geq 2}$ decreases monotonically and that $\lim _{m \rightarrow+\infty} E(m)=0$. It is easy to verify that sequence $\{E(m)\}_{m \in N, m \geq 2}$ satisfies the following recurrence relation:

$$
\begin{gathered}
-2 m E(m)+(2 m+3) E(m+1) \\
=\frac{1}{2 m+1}-\frac{(m+1)(2 m) !}{((m+1) !)^{2} 4^{m}} .
\end{gathered}
$$

Consider the sequence $\{e(m)\}_{m \in N, m \geq 2}$ where

$$
e(m)=g(m) \cdot S(m)
$$

and

$$
S(m)=\sum_{j=1}^{m-1} \frac{(2 j+2) !\left(((2 j) ! !)^{2}(j+1)-(2 j+1) !\right)}{2^{(2 j+3)}(2 j+1)((j+1) !)^{2}((2 j) ! !)^{2}}
$$

and

$$
g(m)=\frac{m !(m-1) ! 2^{(2 m+1)}}{(2 m+1) !} .
$$

It is easy to verify that sequence $\{e(m)\}_{m \in N, m \geq 2}$ satisfies the recurrence relation (49). Given that sequences $\{E(m)\}_{m \in N, m \geq 2}$ and $\{e(m)\}_{m \in N, m \geq 2}$ agree for $m=2$ and $m=3$, we conclude that

$$
E(m)=e(m), \quad \text { for } m \in N, m \geq 2 \text {. }
$$

We prove that sequence $\{e(m)\}_{m \in N, m \geq 2}$ is a monotonically decreasing sequence and $\lim _{m \rightarrow+\infty} e(m)=0$.

By the principle of mathematical induction, it follows that

$$
(2 j+1) !<((2 j) ! !)^{2}(j+1)
$$

is true for all $j \in N$. Therefore $S(m)>0$ for $m \geq 2$, i.e.,

$$
e(m)>0, \quad \text { for } m \geq 2 .
$$

To prove that $\left\{e_{m}\right\}_{m \in N, m \geq 2}$ is a monotonically decreasing sequence, let us use the following notation:

$$
S(m)=\sum_{j=1}^{m-1} h(j)
$$

where

$$
h(j)=\frac{(2 j+2) !\left(((2 j) ! !)^{2}(j+1)-(2 j+1) !\right)}{2^{(2 j+3)}(2 j+1)((j+1) !)^{2}((2 j) ! !)^{2}} .
$$


Consider the following equivalences for $m \geq 2$ :

$$
\begin{array}{r}
\frac{e(m+1)}{e(m)}<1 \Longleftrightarrow \\
\frac{g(m+1) S(m+1)}{g(m) S(m)}<1 \Longleftrightarrow \\
\frac{g(m+1)}{g(m)} \cdot \frac{S(m)+h(m)}{S(m)}<1 \Longleftrightarrow \\
\frac{2 m}{2 m+3} \cdot \frac{S(m)+h(m)}{S(m)}<1 \Longleftrightarrow \\
2 m h(m)<3 S(m) .
\end{array}
$$

Consider the last inequality. It is easy to verify that it is true for $m=2$. Observing that

$$
3 S(m+1)=3(S(m)+h(m))=3 S(m)+3 h(m)
$$

and using the induction hypothesis $(3 S(m)>2 m h(m))$ for some positive integer $m \geq 2$, we conclude that

$$
3 S(m+1)>(2 m+3) h(m)>2(m+1) h(m) .
$$

Therefore, by the principle of mathematical induction, the inequality

$$
2 m h(m)<3 S(m)
$$

is true for $m \geq 2$, i.e.,

$$
\frac{e(m+1)}{e(m)}<1, \text { for } m \geq 2 \text {. }
$$

Let us further consider the positive addend of $S(m)$, i.e.,

$$
\begin{aligned}
S^{+}(m) & =\sum_{j=1}^{m-1} \frac{(2 j+2) !((2 j) ! !)^{2}(j+1)}{2^{(2 j+3)}(2 j+1)((j+1) !)^{2}((2 j) ! !)^{2}} \\
& =\sum_{j=1}^{m-1} \frac{(2 j) !}{4((2 j) ! !)^{2}} .
\end{aligned}
$$

By the principle of mathematical induction, it follows that

$$
S^{+}(m)=\frac{m(2 m) !}{2((2 m) ! !)^{2}}-\frac{1}{4} .
$$

Finally, given that for $m \geq 2$

$$
0<e(m) \leq g(m) \cdot S^{+}(m)=\frac{1}{2 m+1}
$$

we have $\lim _{m \longrightarrow+\infty} e(m)=0$.

Finally, based on (53) we conclude that $\{E(m)\}_{m \in N, m \geq 2}$ is a positive monotonically decreasing sequence and that it tends to zero. The same holds true for the sequence $\left\{E(m) x^{2 m+1}\right\}_{m \in N, m \geq 2}$ for a fixed $x \in(0,1]$ so we can apply Leibniz's theorem for alternating series [28], thus proving the claim of Theorem 10.
2.3. Refinements of the Inequalities in Statement 3. We propose the following improvement and generalization of Statement 3.

Theorem 11. For every $x \in(0,1]$ and $k \in N$, it is asserted that

$$
\begin{aligned}
& \sum_{m=1}^{2 k-1}(-1)^{m} C(m) x^{2 m+1}<\arctan x-\frac{2 x}{1+\sqrt{1+x^{2}}} \\
& \quad<\sum_{m=1}^{2 k}(-1)^{m} C(m) x^{2 m+1},
\end{aligned}
$$

where

$$
C(m)=\frac{1}{2 m+1}-\frac{(2 m-1) ! !}{(m+1) ! 2^{m}}
$$

Examples. For $x \in(0,1]$ and $k=1$ we get inequality (4) from Statement 3.

For $x \in(0,1]$ and $k \geq 2$ inequality (66) from Theorem 11 refines inequality (4) from Statement 3 and we have the following new results:

(i) Taking $k=2$ in (66) gives

$$
\begin{aligned}
&- \frac{1}{12} x^{3}+\frac{3}{40} x^{5}-\frac{29}{448} x^{7}<\arctan x-\frac{2 x}{1+\sqrt{1-x^{2}}} \\
&<-\frac{1}{12} x^{3}+\frac{3}{40} x^{5}-\frac{29}{448} x^{7}+\frac{65}{1152} x^{9} .
\end{aligned}
$$

(ii) Taking $k=3$ in (66) gives

$$
\begin{aligned}
& -\frac{1}{12} x^{3}+\frac{3}{40} x^{5}-\frac{29}{448} x^{7}+\frac{65}{1152} x^{9}-\frac{281}{5632} x^{11} \\
& <\arctan x-\frac{2 x}{1+\sqrt{1-x^{2}}} \\
& <-\frac{1}{12} x^{3}+\frac{3}{40} x^{5}-\frac{29}{448} x^{7}+\frac{65}{1152} x^{9}-\frac{281}{5632} x^{11} \\
& \quad+\frac{595}{13312} x^{13},
\end{aligned}
$$

etc.

Proof of Theorem 11. For $x \in(0,1]$ the following power series expansion holds:

$$
\begin{aligned}
& \arctan x-\frac{2 x}{1+\sqrt{1+x^{2}}} \\
& =\arctan x+\frac{2\left(1-\sqrt{x^{2}+1}\right)}{x} \\
& =\sum_{m=1}^{\infty}(-1)^{m} C(m) x^{2 m+1}
\end{aligned}
$$

where

$$
C(m)=\frac{1}{2 m+1}-\frac{(2 m-1) ! !}{(m+1) ! 2^{m}}
$$


We prove that the sequence $\{C(m)\}_{m \in N}$ is positive and monotonically decreasing and tends to zero as $m$ tends to infinity.

It is easy to verify that $(2 m+2) ! !>2(2 m+1) ! !$ is true for $m \in N$. Thus, the following equivalences hold true for every $m \in N$ :

$$
\begin{aligned}
& C(m)>0 \Longleftrightarrow \\
& \frac{1}{2 m+1}>\frac{(2 m-1) ! !}{(m+1) ! 2^{m}} \Longleftrightarrow \\
& (2 m+2) ! !>2(2 m+1) ! !,
\end{aligned}
$$

and we conclude that $C(m)>0$ for every $m \in N$.

Let us now prove that $\{C(m)\}_{m \in N}$ is a monotonically decreasing sequence. We have

$$
\begin{aligned}
& C(m)-C(m+1)>0 \Longleftrightarrow \\
& \frac{2}{(2 m+1)(2 m+3)}-3 \frac{(2 m-1) ! !}{(2 m+2) ! !(m+2)}>0 \Longleftrightarrow \\
& (2 m+4) ! !>3(2 m+3) ! ! .
\end{aligned}
$$

As it is easy to show (by the principle of mathematical induction) that the last inequality holds true for $m \in N$, we may conclude that $\{C(m)\}_{m \in N}$ is a monotonically decreasing sequence.

Finally, as $0<C(m)<1 /(2 m+1)$, we conclude that $\lim _{m \rightarrow+\infty} C(m)=0$.

Since $\{C(m)\}_{m \in N}$ is a positive monotonically decreasing sequence, and it tends to zero, the same holds true for the sequence $\left\{C(m) x^{2 m+1}\right\}_{m \in N}$ for a fixed $x \in(0,1]$. So we can apply Leibniz's theorem for alternating series [28] and thus prove the claim of Theorem 11.

\section{Conclusion}

In Theorems 9, 10, and 11 of this paper we proved some new inequalities related to Shafer's inequality for the arctangent function. These inequalities represent sharpening and generalization of the inequalities given in [8] (Theorems 1,2, and 4).

Let us mention that it is possible to prove inequality (35), for any fixed $k \in N$ and $x \in(0, \sqrt{3} / 2]$, by substituting $x=$ $\tan t$ for $t \in(0, \pi / 3]$ using the algorithms and methods (see also $[29,30])$ developed in $[31,32]$. Also, inequalities (42) and (66) for any fixed $k \in N$ and $x \in(0,1]$ can be proved by substituting $x=\tan t$ for $t \in(0, \pi / 4]$ using the algorithms and methods (see also $[29,30]$ ) developed in $[31,32]$.

\section{Conflicts of Interest}

The authors would like to state that they do not have any conflicts of interest in the subject of this research.

\section{Authors' Contributions}

All the authors participated in every phase of the research conducted for this paper.

\section{Acknowledgments}

Research of the first, second, and third author was supported in part by the Serbian Ministry of Education, Science and Technological Development, under Projects ON 174032, III 44006, ON 174033, and TR 32023, respectively.

\section{References}

[1] G. T. F. De Abreu, "Jensen-Cotes upper and lower bounds on the Gaussian Q-function and related functions," IEEE Transactions on Communications, vol. 57, no. 11, pp. 3328-3338, 2009.

[2] G. Rahmatollahi and G. Abreu, "Closed-form hop-count distributions in random networks with arbitrary routing," IEEE Transactions on Communications, vol. 60, no. 2, pp. 429-444, 2012.

[3] G. Alirezaei and R. Mathar, "Scrutinizing the average error probability for Nakagami fading channels," in Proceedings of the 2014 IEEE International Symposium on Information Theory, ISIT 2014, pp. 2884-2888, USA, July 2014.

[4] Gabriel Bercu, "Sharp Refinements for the Inverse Sine Function Related to Shafer-Fink's Inequality," Mathematical Problems in Engineering, vol. 2017, Article ID 9237932, 5 pages, 2017.

[5] G. Alirezaei, A sharp double inequality for the inverse tangent function, arXiv:1307.4983, 2013.

[6] G. Alirezaei, Optimizing Power Allocation in Sensor Networks with Application in Target Classification, Dissertation - RWTH Kontinuumsmechanik - RWTH Aachen University, D 82, 1-153, 2014.

[7] M. J. Cloud, B. C. Drachman, and L. Lebedev, Inequalities, Springer, Cham, Second edition, 2014.

[8] C. Mortici and H. M. Srivastava, "Estimates for the arctangent function related to Shafer's inequality," Colloquium Mathematicum, vol. 136, no. 2, pp. 263-270, 2014.

[9] E. O. Thorp, M. Fried, R. E. Shafer et al., "Problems and Solutions: Elementary Problems: E1865-E1874," The American Mathematical Monthly, vol. 73, no. 3, pp. 309-310, 1966.

[10] R. E. Shafer, “On quadratic approximation," SIAM Journal on Numerical Analysis, vol. 11, pp. 447-460, 1974.

[11] R. E. Shafer, "Analytic inequalities obtained by quadratic approximation," Univerzitet u Beogradu. Publikacije Elektrotehničkog Fakulteta. Serija Matematika i Fizika, no. 577-598, pp. 96-97, 1977.

[12] R. E. Shafer, “On quadratic approximation II," Univerzitet $u$ Beogradu. Publikacije Elektrotehničkog Fakulteta. Serija Matematika i Fizika, no. 602-633, pp. 163-170 (1979), 1978.

[13] L. Zhu, "On a quadratic estimate of Shafer," Journal of Mathematical Inequalities, vol. 2, no. 4, pp. 571-574, 2008.

[14] L. Zhu, "A source of inequalities for circular functions," Computers \& Mathematics with Applications, vol. 58, no. 10, pp. 19982004, 2009.

[15] F. Qi, D. Niu, and B. Guo, "Refinements, Generalizations, and Applications of Jordan's Inequality and Related Problems," Journal of Inequalities and Applications, vol. 2009, Article ID 271923, 52 pages, 2009.

[16] F. Qi, S. Zhang, and B. Guo, "Sharpening and Generalizations of Shafer's Inequality for the Arc Tangent Function," Journal of Inequalities and Applications, vol. 2009, Article ID 930294, 9 pages, 2009. 
[17] J. D’Aurizio, "Refinements of the Shafer-Fink inequality of arbitrary uniform precision," Mathematical Inequalities \& Applications, vol. 17, no. 4, pp. 1487-1498, 2014.

[18] B. A. Bhayo and J. Sandor, “On Carlson's and Shafer's inequalities," Problemy Analiza. Issues of Analysis, vol. 3(21), no. 1, pp. 3-15, 2014.

[19] J.-L. Sun and C.-P. Chen, "Shafer-type inequalities for inverse trigonometric functions and Gauss lemniscate functions," Journal of Inequalities and Applications, Paper No. 212, 9 pages, 2016.

[20] S. Wu and G. Bercu, "Pade approximants for inverse trigonometric functions and their applications," Journal of Inequalities and Applications, Paper No. 31, 12 pages, 2017.

[21] Y. Nishizawa, "Refined quadratic estimations of Shafer's inequality," Journal of Inequalities and Applications, Paper No. 40, 11 pages, 2017.

[22] D. S. Mitrinović and P. M. Vasić, Analytic Inequalities, Springer, Berlin, Germany, 1970.

[23] G. V. Milovanović and M. T. Rassias, Eds., Analytic Number Theory, Approximation Theory and Special Functions, Springer, Chapter: G. D. Anderson, M. Vuorinen, X. Zhang: Topics in Special Functions III, pp. 297-345, 2014.

[24] M. Petkovšek, H. S. Wilf, and D. Zeilberger, “A=B, A. K. Peters," Wellesley, 1996.

[25] A. Tefera, "What is a Wilf-Zeilberger pair?" Notices Of the American Mathematical Society, vol. 57, no. 4, pp. 508-509, 2010.

[26] M. Petkovšek and H. Zakrajšek, Solving Linear Recurrence Equations with Polynomial Coefficients, Computer Algebra in Quantum Field Theory, Springer, 2013.

[27] B. Malešević, M. Rašajski, and T. Lutovac, "Refinements and generalizations of some inequalities of Shafer-Fink's type for the inverse sine function," Journal of Inequalities and Applications, Paper No. 275, 9 pages, 2017.

[28] R. Beals, Analysis - an introduction, Cambridge University Press, 2004.

[29] B. Malešević, T. Lutovac, and B. Banjac, "A proof of an open problem of Yusuke Nishizawa for a power-exponential function," Journal of Mathematical Inequalities, vol. 12, no. 2, pp. 473485, 2018.

[30] B. Malešević, B. Banjac, and I. Jovović, "A proof of two conjectures of Chao-Ping CHEn for inverse trigonometric functions," Journal of Mathematical Inequalities, vol. 11, no. 1, pp. 151-162, 2017.

[31] B. Malešević and M. Makragić, "A method for proving some inequalities on mixed trigonometric polynomial functions," Journal of Mathematical Inequalities, vol. 10, no. 3, pp. 849-876, 2016.

[32] T. Lutovac, B. Malešević, and C. Mortici, "The natural algorithmic approach of mixed trigonometric-polynomial problems," Journal of Inequalities and Applications, Paper No. 116, 16 pages, 2017. 


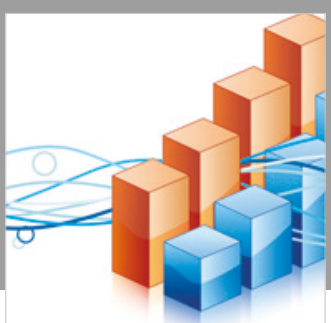

Advances in

Operations Research

\section{-n-m}
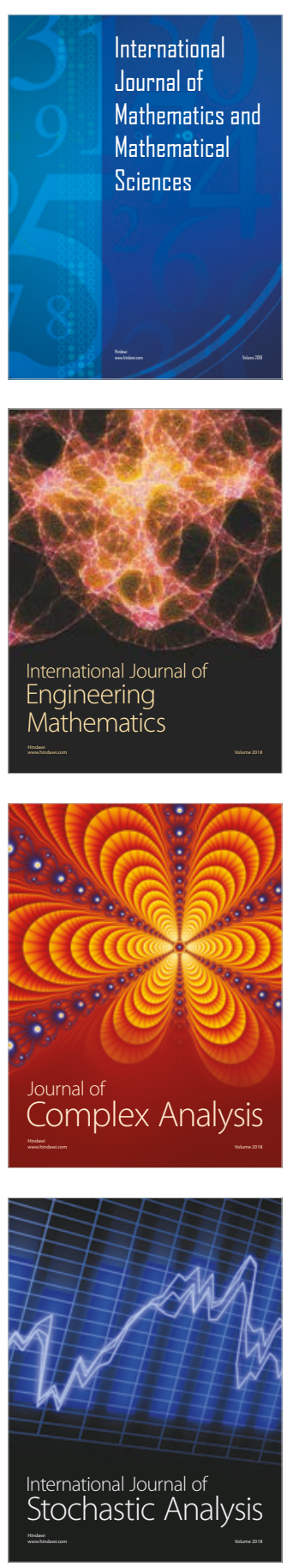
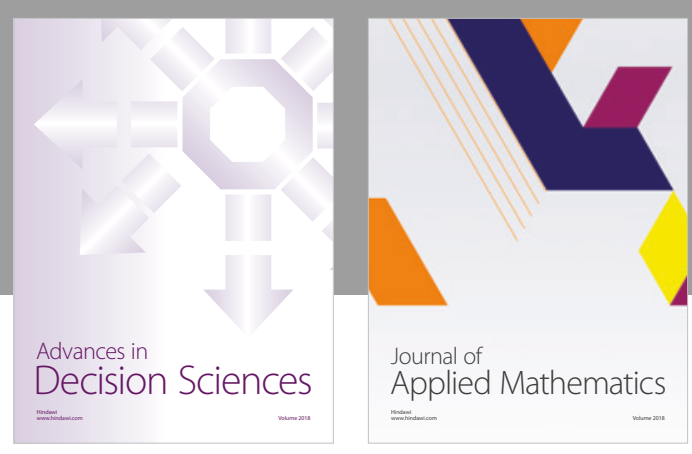

Journal of

Applied Mathematics
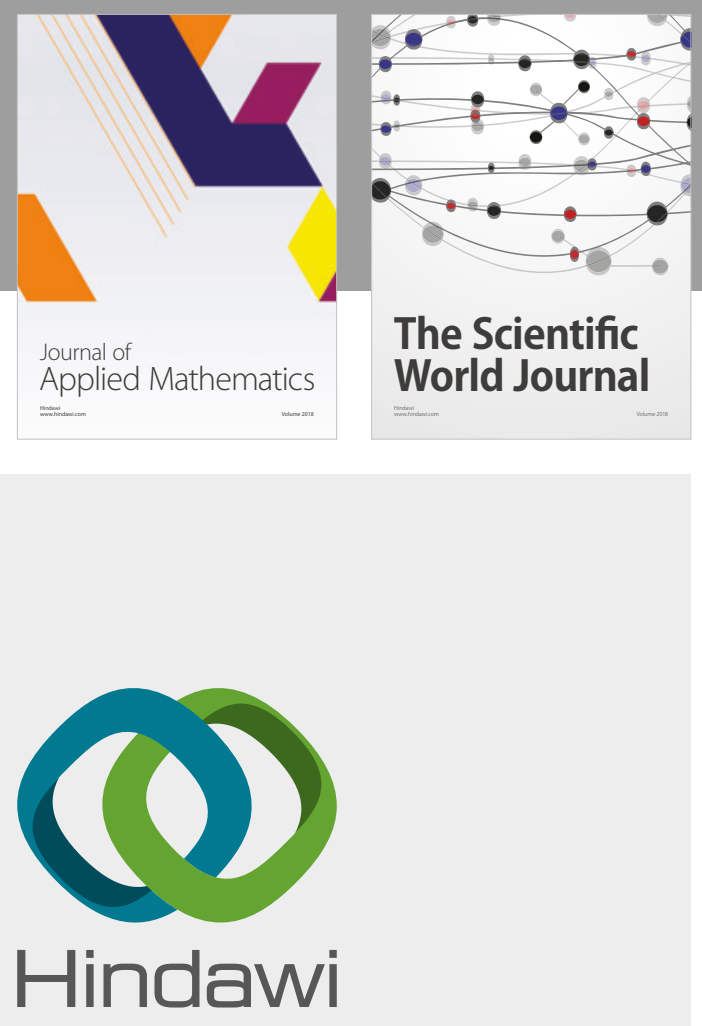

Submit your manuscripts at

www.hindawi.com

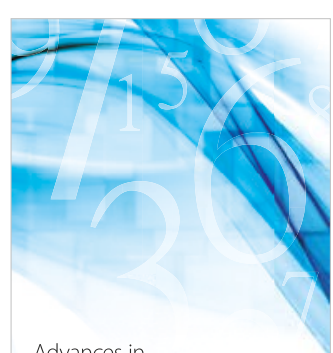

Advances in
Numerical Analysis
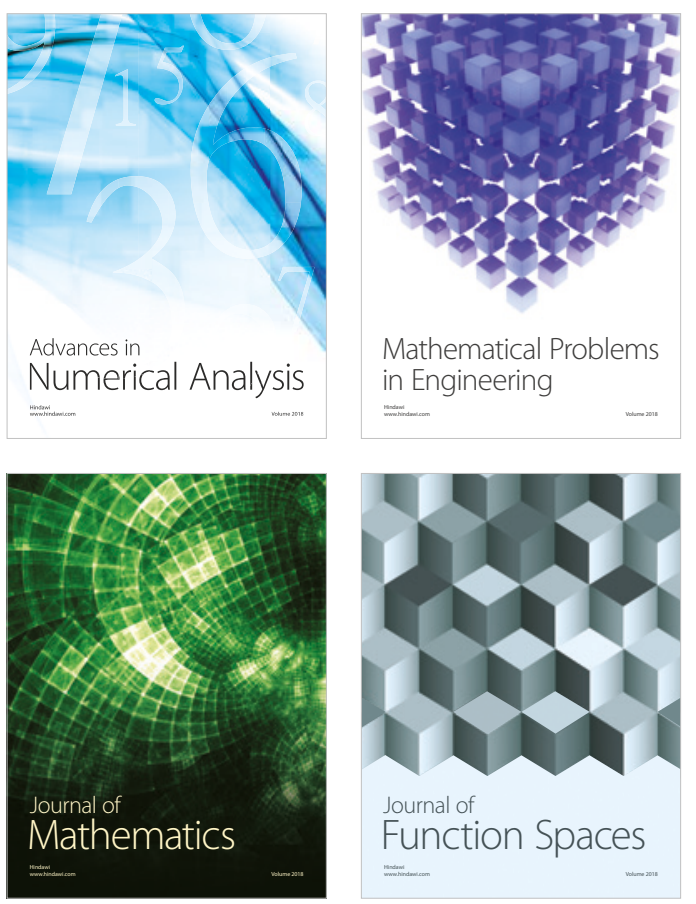

Mathematical Problems in Engineering

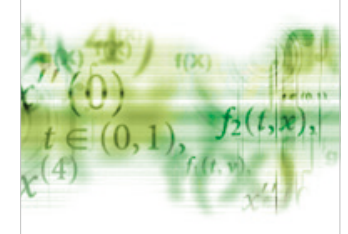

International Journal of

Differential Equations

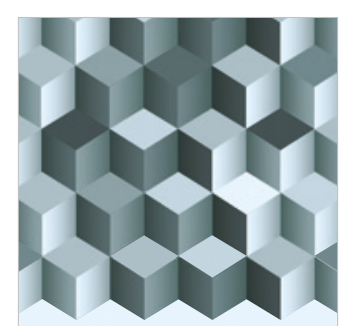

Journal of

Function Spaces

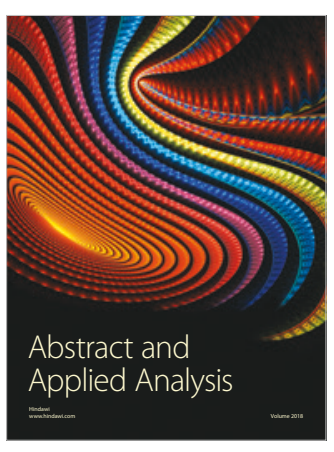

The Scientific

World Journal

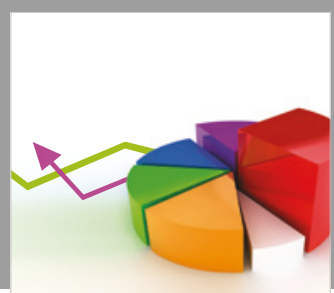

Journal of

Probability and Statistics
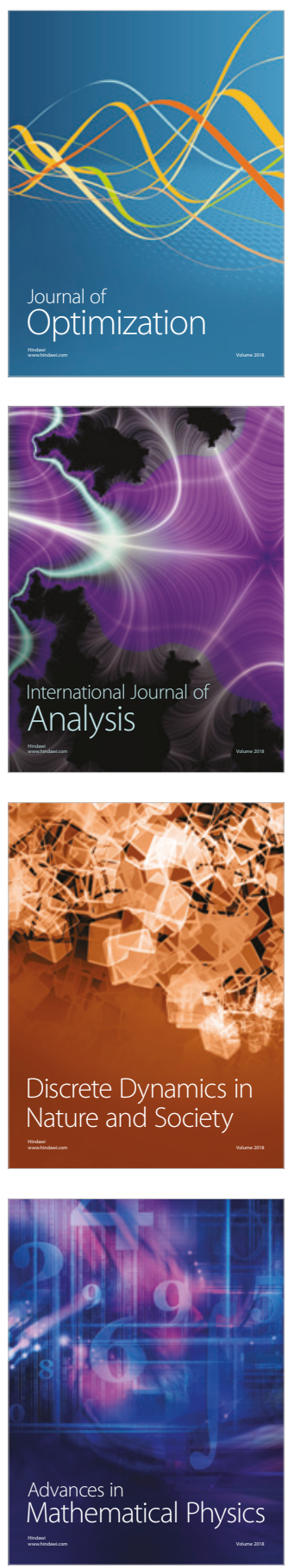